\title{
Effect of the Oxide-Carbon Heterointerface on the
}

\author{
Activity of $\mathrm{Co}_{3} \mathrm{O}_{4} / \mathrm{NRGO}$ Nanocomposites \\ Towards ORR and OER
}

Kavita Kumar, Christine Canaff, Julie Rousseau, Sandrine Arrii-Clacens, Têko W. Napporn, Aurélien Habrioux*, Kouakou B. Kokoh*

Université de Poitiers, IC2MP UMR-CNRS 7285, 4 rue Michel Brunet-B27, TSA 51106, 86073 Cedex 9, France 


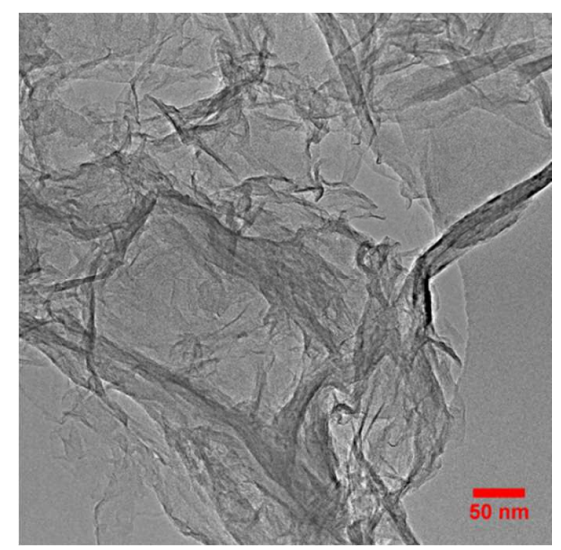

Figure S1. TEM images obtained during the observation of reduced graphene oxide

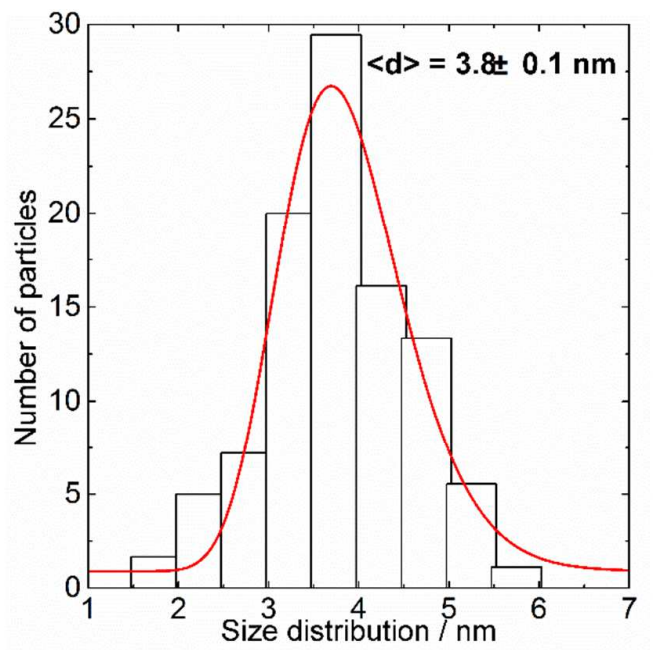

Figure S2. Particle size distribution of $\mathrm{Co}_{3} \mathrm{O}_{4}$ nanoparticles in $\mathrm{Co}_{3} \mathrm{O}_{4} / \mathrm{NRGO}$ hybrid

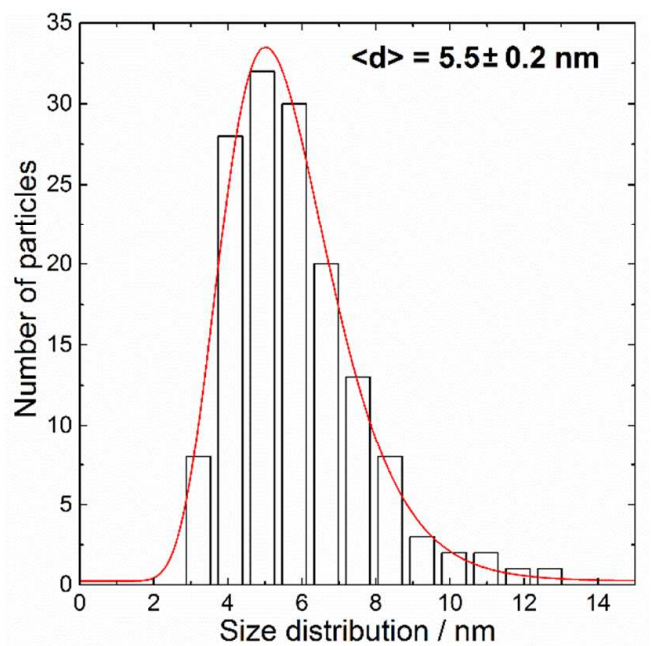

Figure S3. Particle size distribution of $\mathrm{Co}_{3} \mathrm{O}_{4}$ nanoparticles in $\mathrm{Co}_{3} \mathrm{O}_{4} / \mathrm{RGO}$ hybrid 


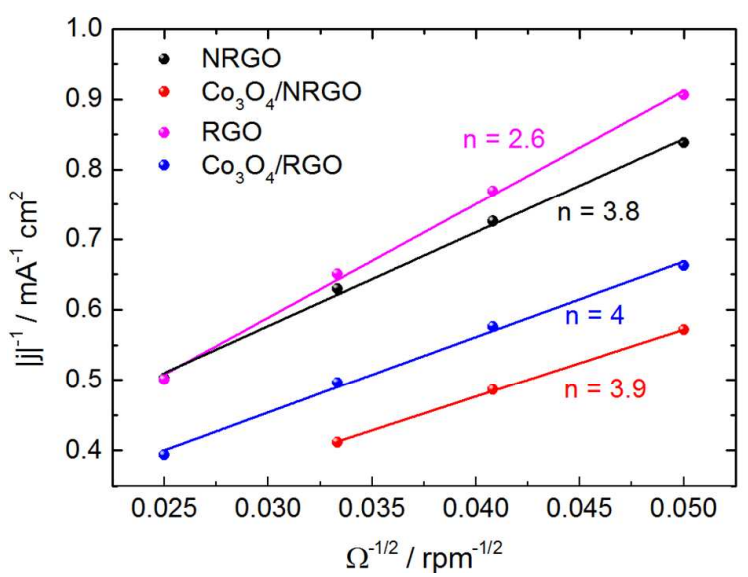

Figure S4. Koutecky-Levich plots obtained from ORR polarization curves recorded using $\mathrm{RGO}, \mathrm{NRGO}, \mathrm{Co}_{3} \mathrm{O}_{4} / \mathrm{RGO}$ and $\mathrm{Co}_{3} \mathrm{O}_{4} / \mathrm{NRGO}$ materials as catalysts

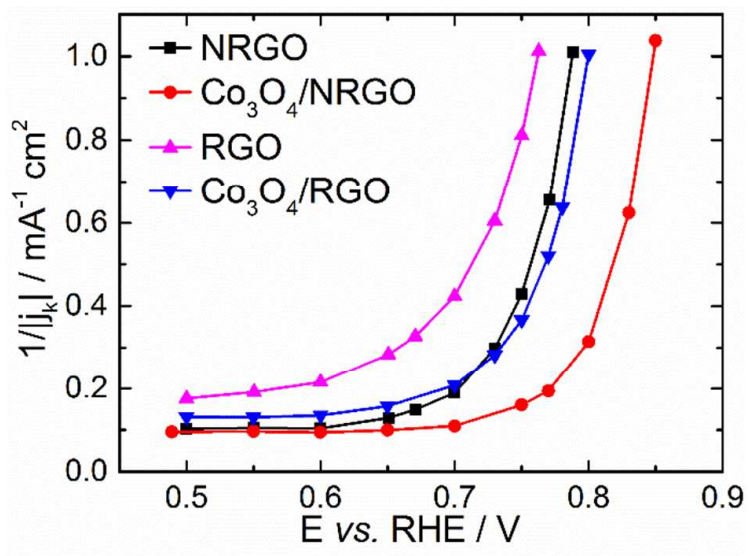

Figure S5. Variation of $1 /\left|j_{k}\right| v s$. the potential, obtained from the Koutecky-Levich treatment using RGO, $\mathrm{NRGO}, \mathrm{Co}_{3} \mathrm{O}_{4} / \mathrm{RGO}$ and $\mathrm{Co}_{3} \mathrm{O}_{4} / \mathrm{NRGO}$ materials as catalysts

Table S6. Electrical performances of bifunctional catalysts from literature.

\begin{tabular}{cccc} 
Catalysts & Oxide mass loadinga $(\%)$ & $\Delta \mathbf{E}^{\mathbf{b}}(\mathbf{m V})$ & Ref \\
$\mathrm{Co}_{3} \mathbf{O}_{4} / \mathbf{N R G O}$ & 30 & 810 & this work \\
$\mathrm{Co}_{3} \mathbf{O}_{4} / \mathbf{N}-\mathbf{r m G O}$ & 70 & 710 & 1 \\
$\mathrm{MnCo}_{2} \mathbf{O}_{4} / \mathbf{N}-\mathbf{r m G O}$ & 80 & 730 & 2 \\
$\mathbf{N i C o}_{2} \mathbf{O}_{4}-\mathbf{G}$ & - & 900 & 3 \\
$\mathbf{C o F e}_{2} \mathbf{O}_{4} / \mathbf{r G O}$ & 70 & 918 & 4 \\
\hline
\end{tabular}

${ }^{\text {a }}$ Oxide mass loading on graphene supported, determined by TGA. 
${ }^{\mathrm{b}} \Delta \mathrm{E}$ : Potential difference: $\mathrm{E}_{\mathrm{j} 10}-\mathrm{E}_{\mathrm{j} 1}$ where $\mathrm{E}_{\mathrm{j} 1}$ is the potential required for a current density of 1 $\mathrm{mA} \mathrm{cm}{ }^{-2}$ during the ORR and $E_{j 10}$ the potential required for a current density of $10 \mathrm{~mA} \mathrm{~cm} \mathrm{~cm}^{-2}$ during the OER.

(1) Liang, Y.; Li, Y.; Wang, H.; Zhou, J.; Wang, J.; Regier, T.; Dai, H., Co3o4 Nanocrystals on Graphene as a Synergistic Catalyst for Oxygen Reduction Reaction. Nat Mater 2011, 10, 780-786.

(2) Liang, Y.; Wang, H.; Zhou, J.; Li, Y.; Wang, J.; Regier, T.; Dai, H., Covalent Hybrid of Spinel Manganese-Cobalt Oxide and Graphene as Advanced Oxygen Reduction Electrocatalysts. J. Am. Chem. Soc. 2012, 134, 3517-3523.

(3) Lee, D. U.; Kim, B. J.; Chen, Z., One-Pot Synthesis of a Mesoporous Nico2o4 Nanoplatelet and Graphene Hybrid and Its Oxygen Reduction and Evolution Activities as an Efficient Bi-Functional Electrocatalyst. J. Mater. Chem. A 2013, 1, 4754-4762.

(4) Bian, W.; Yang, Z.; Strasser, P.; Yang, R., A $\mathrm{CoFe}_{2} \mathrm{O}_{4} /$ Graphene Nanohybrid as an Efficient Bi-Functional Electrocatalyst for Oxygen Reduction and Oxygen Evolution. J. Power Sources 2014, 250, 196-203. 